\title{
Deficits in plasma oxytocin responses and increased negative affect, stress, and blood pressure in mothers with cocaine exposure during pregnancy
}

\author{
Kathleen C. Light ${ }^{\mathrm{a},{ }^{*}}$, Karen M. Grewen ${ }^{\mathrm{a}}$, Janet A. Amico ${ }^{\mathrm{b}}$, Maria Boccia ${ }^{\mathrm{c}}$, Kimberly A. \\ Brownley $^{a}$, and Josephine M. Johns ${ }^{a}$ \\ a Department of Psychiatry, School of Medicine, University of North Carolina, CB \#7175 Medical \\ Building A, Chapel Hill, NC 27599-7175, USA \\ b Departments of Medicine and Pharmaceutical Sciences, University of Pittsburgh, Pittsburgh, PA \\ 15261, USA \\ ${ }^{c}$ Frank Porter Graham Child Development Institute, University of North Carolina, Chapel Hill, NC \\ 27599, USA
}

\begin{abstract}
In animals, oxytocin enhances maternal behavior and lowers blood pressure (BP) and negative affect, while parturitional cocaine disrupts oxytocin activity and increases maternal neglect and aggression. Thus, we compared oxytocin, BP, maternal behavior, and affect in mothers of infants who used cocaine (cocaine, $n=10$ ) or did not (no drug, $n=25$ ) during pregnancy. Laboratory BP and circulating oxytocin, catecholamines, and cortisol were examined before and during a speech stressor on 2 days, with vs. without prestress baby holding. Ambulatory monitoring assessed BP, urinary norepinephrine, and cortisol for $24 \mathrm{~h}$ at home. The cocaine group had lower oxytocin levels, greater hostility and depressed mood, less support from others and mastery over life events, higher BP during all events of testing without the baby, and higher ambulatory BP and urinary norepinephrine at home, while cortisol and epinephrine responses were blunted. Although they tended to hold their babies less often at home, baby holding in the laboratory led to decreased BP in cocaine mothers who then did not differ from no-drug mothers in BP or observed affect.
\end{abstract}

\section{Keywords}

Cocaine exposure; Blood pressure; Plasma oxytocin responses; Maternal-infant interaction

\section{Introduction}

As primary caregivers, mothers who abuse drugs during pregnancy are at high risk for problems in parenting skills (Lawson \& Wilson, 1980). A history of substance abuse has been associated with poor maternal-infant interactions (Bauman \& Dougherty, 1983; Bays, 1990; Howard, Beckwith, Espinosa, \& Tyler, 1995; Johnson \& Rosen, 1990), as well as inadequate social support systems and low self-esteem and maternal ego development (Fineman, Beckwith, Howard, \& Espinosa, 1997; Williams-Petersen et al., 1994).

Specifically, maternal cocaine abuse during pregnancy has been correlated with a greater incidence of maternal neglect (Kelly, Walsh, \& Thompson, 1992), problems with maternal-

(C) 2004 Elsevier Ltd. All rights reserved.

*Corresponding author. Tel.: +1-919-9662544; fax: +1-919-966-0708. kalight@med.unc.edu (K.C. Light). 
infant bonding (Burns, Chethik, Burns, \& Clark, 1991), and child abuse in the human population (Murphy et al., 1991). A relatively recent study found that women who had taken cocaine during pregnancy had more difficulty interacting with their infants and expressed subtle hostility during feeding and play periods as compared to nondrug-abusing women (Goldman-Fraser, 1997).

In an animal model of maternal neglect, it was found that exposure to cocaine in pregnant rats decreases their subsequent maternal behavior and increases inappropriate and nonadaptive aggressive behavior. Specifically, rats given a moderate dose of cocaine either acutely during the postpartum period or chronically during pregnancy display increased maternal neglect of offspring (less feeding, licking, and nestbuilding) and either compulsive nonadaptive aggressive behavior (after chronic gestational treatment) or nonprotective behavior (after acute cocaine postpartum treatment) in the presence of an intruder (Johns et al., 1998; Johns, Noonan, Zimmerman, Li, \& Pedersen, 1994). Continuing exposure to postpartum cocaine may result in somewhat different patterns of disruptions in maternal behavior or aggression depending on dose and frequency of use during pregnancy (Johns, Lubin, Walker, Meter, \& Mason, 1997; Johns, Noonan, Zimmerman, Li, \& Pedersen, 1997; Johns et al., 2002; Nelson, Meter, Walker, Ayers, \& Johns, 1998). These abnormal maternal behaviors appeared to be at least partially mediated by cocaine-induced changes in oxytocin activity (Elliott, Lubin, \& Johns, 2001; Johns, Lubin et al., 1997; Lubin, Elliott, Black, \& Johns, 2003).

The neuropeptide, oxytocin, is linked in animal models to both initiation and perhaps early maintenance of maternal behaviors, including licking and nursing (Champagne \& Meaney, 2001; Johns, Lubin et al., 1997; Pedersen, Caldwell, Walker, Ayers, \& Mason, 1994), and to antistress and blood pressure (BP) lowering effects (Knox \& Uvnas-Moberg, 1998; Petersson, Alster, Lundeberg, \& Uvnas-Moberg, 1996). Oxytocin administration to female sheep will induce them to adopt and nurse orphan lambs, demonstrating oxytocin's role in maternal-offspring bonding (Keverne \& Kendrick, 1992). In rats, exogenous oxytocin or endogenous oxytocin activity elicited by stroking gives rise to antistress effects [e.g., decreases in activity of the hypothalamic-pituitary-adrenal (HPA) axis and BP, but greater stress analgesia] after repeated administration (Holst, Uvnas-Moberg, \& Petersson, 2002; Petersson, Uvnas-Moberg, Erhardt, \& Engberg, 1998; Robinson et al., 2002). Preliminary evidence from human mothers suggests that oxytocin helps modulate their affective state, and this may, in turn, influence their stress responses. In mothers who both breast- and bottle-feed their infants, the act of breast-feeding (which typically elicits greater rises in plasma oxytocin) is associated with less perceived stress, depression, and anxiety than bottle-feeding (Heck \& de Castro, 1993; Mezzacappa \& Katkin, 2002; Modahl \& Newton, 1979). Furthermore, women who are breast-feeders also report less anxiety than bottlefeeding women and show lower BP before and during stress (Altemus et al., 2001; Mezzacappa \& Katkin, 2002; Virden, 1988). However, oxytocin responses of lactating and nonlactating women do not consistently differ (Altemus et al., 2001), and even among breast-feeders, there are individual differences in oxytocin responses that directly relate to affective state. Uvnas-Moberg, Widstrom, Nessen, and Bjorvell (1990) reported that baseline oxytocin levels in breast-feeding women were lower in those scoring high in anxiety, aggression, guilt, and suspicion. Our own findings in mothers of infants suggest that increased BP (both during laboratory and home monitoring) and higher negative affect are seen in mothers with low plasma oxytocin responsivity (Light, Smith, Johns, Hofheimer, \& Amico, 2001). In this study, oxytocin responses in high responder mothers were enhanced after 10 min of baby holding, cuddling, and play compared to responses of the same women when tested on another day after 10 min resting alone without their babies. Similarly, BP levels at home were lower for an hour after baby holding and nursing in these high oxytocin responding mothers. Together, these observations suggest that in addition to breast-feeding, 
baby holding and cuddling can increase oxytocin activity in more responsive mothers, and this may be associated with decreases in BP and more positive mood states. Conversely, low oxytocin responses may be related to increased BP, negative emotions, and stress sensitivity in mothers of infants.

Although there is some evidence that cocaine use during pregnancy may be associated with more negative affect, hostile or aggressive behavior, and maternal neglect, no studies to date have compared oxytocin responses in mothers who used cocaine during pregnancy vs. those who did not, nor have any studies addressed the effects of recent baby holding and cuddling on these mothers. Thus, the present investigation was designed to test the following hypotheses. (1) Mothers who were (vs. were not) exposed to cocaine during pregnancy show lower plasma oxytocin levels and reduced oxytocin responses during stress. (2) The cocaineexposed mothers also show less maternal behavior at home (decreased time at home spent holding their baby) and in the laboratory (less positive and more negative affect during baby holding and play), report greater hostility, depression, anxiety and recent life stress, and exhibit higher BP, cortisol, and norepinephrine levels both in the laboratory and their home environments. (3) Stress testing immediately after baby holding and cuddling will be associated with greater plasma oxytocin responses, lower norepinephrine, and lower BP than testing without prior baby contact, and these effects will be greater among mothers with no cocaine exposure during pregnancy.

\section{Methods}

\subsection{Participants}

Participants in this study included 25 recent mothers (at least 1 month but not more than 11 months postpartum) who had no history during pregnancy or postpartum of exposure to cocaine or other substances of abuse, and 10 recent mothers who reported using cocaine at least once during their pregnancy. All of the cocaine-exposed mothers were recruited through a local substance abuse rehabilitation and support program for pregnant women, focusing on intensive prenatal and postnatal care for mothers with substance-abuse problems. All had been participants for 60 days or longer in this rehabilitation program which required them to refrain from using cocaine or other substances of abuse, confirmed by regular urine screens. Thus, our observations should not be influenced by recent drug use. All of the mothers in the cocaine-exposed group were bottle-feeders; this feeding option was strongly encouraged by medical and social services personnel to ensure that the infants would not receive any postnatal substance exposure. To try to recruit no-drug mothers who were also receiving similar public assistance, we recruited other mothers through a statewide, county-managed nutrition program, focused on meeting nutritional needs of lowincome mothers and children. However, because the nutrition-program mothers were strongly encouraged to breast-feed, and none recruited through this agency was a bottlefeeder, it became necessary to recruit bottle-feeding mothers through special targeted newspaper ads. Mothers in the no-drug group thus included 14 who breast-fed with occasional use of expressed breast milk and 11 who exclusively bottle-fed using formula over the past 30 days. Women who currently used a combination of feeding methods were excluded from participation.

All research information on substance use or lack of it was derived from phone and inperson interview questions, not medical records or urine tests. Mothers in both the no-drug and the cocaine groups were asked during phone screening if they had used any drugs (prescription, over the counter, or recreational) during or since their pregnancy. Marijuana, cocaine, and crack were asked about individually after the general question on substance use. All no-drug mothers stated they had not used any recreational or prescription drugs during this period, while mothers in the cocaine group all stated that they had used cocaine 
or crack. Participants were also asked about use of alcohol, which a similar number of mothers in both groups told us they had used, although all use was limited to one drink per day. Mothers in both groups were asked a second time about any recent use of cocaine or other recreational drugs other than alcohol when they arrived for screening and testing, and all denied any use since the baby's birth. As part of our confidentiality assurance plan approved by the IRB and the special programs referring these mothers, no effort was made to question the mothers in any detail about the number of times or amounts of cocaine used by these mothers during pregnancy. Because all babies were full term and none had low birthweight or other peri- or postnatal complications, it is assumed that these mothers and their babies had relatively low drug exposure. In addition, as part of our agreement to ensure privacy and confidentiality, we did not approach the mothers in either of these programs ourselves; information sheets describing the study were provided to the staff members who worked for these programs for them to distribute to potential volunteers at their discretion. Thus, we have no data on what percentage of those who were eligible agreed to participate, although we do know that of those mothers who subsequently contacted us and who met study criteria, over $90 \%$ were tested. Moreover, as part of the agreement with the referring program, mothers from these programs were compensated for participation with vouchers for purchasing diapers, baby clothing, and other infant care essentials at a local store.

Only mothers of healthy, full-term infants were included, and medical history information was used to exclude women with average resting SBP and DBP >160/95 mm Hg, or any other serious cardiovascular, renal, or pulmonary disorder, or current clinical depression or other psychiatric disorder, or currently using antihypertensive, antidepressant, or other prescription medications.

\subsection{Protocol}

Data from the mothers with no prenatal use of drugs has been reported previously, including details of the protocol and procedures (see Light et al., 2001). In brief, each participant attended a screening session was fully informed and provided written consent for this IRBapproved protocol. Then, in a counterbalanced order, each was tested on two consecutive mornings at the same hour (typically between 9 and 10 a.m.), with one session including a 10-min period of baby contact (holding, reading to, and playing with the baby) and the other session requiring the mother not to bring the baby to the laboratory and involving a restalone control period instead. In all other ways, the sessions were identical, beginning with instrumentation with the Accutracker II ambulatory BP monitor (Suntech, Raleigh, NC) to record BP, then including a 12-min baseline rest, followed by the baby contact or no baby rest period. Then the baby (if present) was taken from the room by a staff member, and the mother performed a speech stressor, in which she was tape recorded as she described a recent event that made her feel angry or stressed. The task began with instructions, followed by 2 min of silent speech preparation, then 3 min of active speech, followed by $3 \mathrm{~min}$ of listening to replay of the recorded speech, while instructed to relax and recover but also to think about how effectively she had explained her reasons for feeling angry or upset.

During the 10-min period of baby contact, mothers and infants were videotaped first as they interacted in response to the instruction "hold and play with your baby as you normally do," and then in a more structured form of play involving a picture book (The Little Book of Hugs by S. Weisinger), depicting children being hugged in different ways with simple text (e.g., "There are daddy hugs." "There are grandma hugs."). These videotapes were replayed and rated by a single blind rater using the 'modified frequency' sampling method, coding every $6 \mathrm{~s}$ of the tape for the presence or absence of the following behaviors (Martin \& Bateson, 1993). The mothers' positive and negative affective behaviors included the following: smile, laugh, frown, kiss, eye contact with baby, talk positive, talk negative, talk neutral, and baby maintenance (diaper check or change, burp, give pacifier, or other sucking item, etc.). 
Because of wide disparities across infants in their stages of development due to age, the babies' behaviors that were recorded included only two: crying and laughing. Because educational differences may have affected responses during the book reading, only responses during first $3 \mathrm{~min}$ of the holding and free play were analyzed; thus, the possible range of scores for each behavior are from 0 (never present) to 30 (present in every 6-s interval).

For the day spent at home between the two test sessions, the mothers continued to wear the ambulatory BP monitor, programmed to take readings 4 times $/ \mathrm{h}$ prior to bedtime and 2 times/h during sleep. For each reading, the mother recorded her activities, indicating whether she had nursed, bottle-fed, or held the baby since the last reading. For purposes of analysis, readings were grouped into sleep, postfeeding (including all readings during and up to $60 \mathrm{~min}$ after breast- or bottle-feeding), and all other waking activities. Mothers also collected a 24-h urine sample for this same day between sessions, which was measured and aliquotted for determination of 24-h urinary norepinephrine and cortisol levels. Compliance with the 24-h urine collection was ascertained using race- and gender-specific normal ranges for 24-h creatinine clearance.

\subsection{Cardiovascular, urine, and blood sampling}

BP measurements were obtained during Min 2, 4, 6, 8, 10, and 12 of the resting baseline and during Min 1 and 3 of the preparation, active speech, and replay/recovery periods. Any reading affected by movement artifact was deleted. Mean baseline levels were defined as the average of all six samples obtained during that period.

Blood samples were obtained for plasma oxytocin and plasma catecholamine levels at the end of baseline and during Min 2 of active speech. The samples were drawn into chilled tubes containing sodium heparin, kept on ice, and cold centrifuged to separate plasma within a few minutes after collection. Plasma was rapidly frozen and maintained at $-80{ }^{\circ} \mathrm{C}$ until assayed. Serum was also obtained at baseline only for determination of serum cortisol. This sample was drawn into a tube, allowed to clot for $30 \mathrm{~min}$, then separated serum was likewise frozen at $-80{ }^{\circ} \mathrm{C}$ until assayed.

Oxytocin was first extracted from plasma and then assayed using a specific oxytocin antibody (Pittsburgh Antibody 2) by Dr. Janet Amico of the University of Pittsburgh following previously published techniques (Amico et al., 1985; Amico, Johnston, \& Vagnucci, 1994). The standard curve was linear between 0.5 and $10 \mathrm{pg} / \mathrm{tube}$, and any sample less than $0.5 \mathrm{pg}$ was designated as zero. Intra- and interassay coefficients of variation were $6.5 \%$ and $8 \%$ respectively; results are reported corrected for recovery (which is $75 \pm 3 \%$ ).

Plasma epinephrine and norepinephrine as well as 24-h urinary norepinephrine levels were determined using high-performance liquid chromatography (HPLC) methods, while serum cortisol and 24-h urinary cortisol levels were determined using radioimmunoassay (RIA) kits commercially available from ICN Biomedical. All assays were performed by trained technicians at the UNC General Clinical Research Center as previously described (Girdler et al., 2003; Koo-Loeb, Costello, Light, \& Girdler, 2000).

Because pretests demonstrated that oxytocin, cortisol, and catecholamine responses from the baseline blood sample drawn on the first day were higher than on the second day, probably due to greater anxiety on the first test day, a single baseline sample averaged across both days was used in all analyses to represent resting levels. 


\subsection{Affect and perceived life stress assessments}

At home prior to testing, participants completed a set of questionnaires focusing on affect and life stress. These included the Beck Depression Inventory (BDI; Beck, Ward, Mendelson, Mock, \& Erbaugh, 1961), a hostility scale consisting of the 15 items (Ho-15) that make up the hostile affect, aggressive responding, and cynical hostility subscales of the 27-item Cook Medley Hostility Scale (Barefoot, Dodge, Peterson, Dahlstrom, \& Williams, 1989; Barefoot, Larsen, von der Lieth, \& Schroll, 1995; Beckham, Calhoun, Glenn, \& Barefoot, 2002; Cook \& Medley, 1954), the Anger Expression Inventory (AX; Spielberger \& Syderman, 1994), the Interpersonal Support Evaluation List (ISEL; Brookings \& Bolton, 1988; Cohen, 1985), the Pearlin Mastery Scale (Goldenberg et al., 1997; Skaff, Pearlin, \& Mullan, 1996), and to assess stress over the past month, the Perceived Stress Scale (Cohen, Kamarck, \& Mermelstein, 1983). On the day of the ambulatory monitoring, they also completed the state and trait subscales of the State-Trait Anxiety Index (STAI; Spielberger, 1983) and, to assess that day's stress, the Daily Stress Inventory (Brantley, Waggoner, Jones, \& Rappaport, 1987).

\subsection{Data analysis}

Demographic information on the mothers is depicted in Table 1. Cocaine and no-drug groups did not differ in mothers' age, body mass index, number of hours spent sleeping on the ambulatory monitoring day, or current use of alcoholic beverages, but did differ in racial distribution $(80 \%$ vs. $31 \%$ black, $P<.005)$, feeding method $(P<.001)$, and education $(50 \%$ vs. $100 \% \geq$ high-school completion, $P<.001$ ), and despite similarities in the range of baby ages included in both groups, they were marginally different in mean baby age ( 3.5 vs. 5.5 months, $P<.10$ ). For all physiological variables involving repeated measurement (effect of baby vs. no baby day for laboratory BP, oxytocin and catecholamine measures, and effect of activity for the ambulatory BP measures), initial analyses using repeated-measures analyses of variance (ANOVAs; PROC GLM, SAS Institutes, Cary, NC) preceded all examinations of univariate analyses to compensate for the increased likelihood of obtaining false positives that may be associated with multiple comparisons. Only when a main effect of group or group $\times$ time period interaction was obtained were univariate analyses examined. In the primary analyses comparing all no-drug vs. cocaine-exposed mothers, feeding method, race, education, and baby age were employed in initial models as covariates, but were retained in final models only when significant. In secondary post hoc analyses separating the no-drug mothers into breast-feeders vs. bottle-feeders, these two groups were compared with each other, as well as being separately compared with the cocaine group. Final contrasts were performed using least-squares means, and this was the primary analysis method for the videotape behavior ratings. Correlations among the multiple measures were examined using simple regression. Unless otherwise indicated, all tests were two-tailed and $\alpha$ was set at $P<$. 05 .

\section{Results}

\subsection{BP responses in the lab}

Repeated-measures ANOVAs yielded significant interaction effects of group $\times$ baby vs. no baby day for DBP, $F(1,34)=4.62, P \leq .04$, and marginally significant interaction effects for SBP, $F(1,34)=3.74, P \leq .06$. Thus, simple effects analyses were performed, comparing SBP and DBP responses between the two groups on each day separately, and also looking within each group for BP differences between the two test days.

On the no baby day, the cocaine-exposed mothers showed higher overall BP levels compared to the no-drug mothers (see Figs. 1 and 2). The cocaine group showed significantly higher DBP at baseline than the no-drug group $(74.0$ vs. $67.3, P<.026)$ as well 
as marginally higher SBP at baseline (110.3 vs. 103.6, $P<.075)$. These group differences were maintained or even enhanced during speech preparation (SBP: 118.7 vs. $108.8, P<$. 034; DBP: 79.5 vs. $71.8, P<.007$ ) and postspeech recovery (SBP: 120.1 vs. $109.9, P<$. 039; DBP: 78.9 vs. $71.8, P<.066$ ) on the no baby day. Differences during active speech were small and did not approach significance.

On the baby contact day, these group differences in BP were no longer present. When the cocaine mothers brought their infants to the study and held and played with them, their BP levels were reduced to levels similar to those of the no-drug mothers and the two groups no longer differed significantly (see Figs. 1 and 2). Most importantly, simple effects analyses comparing SBP and DBP over days within each group separately showed that the cocaine group demonstrated lower DBP across all events on the baby contact day than they showed on the no baby day $(P<.05$ at baseline and during speech preparation, $P=.07$ during active speech and replay), while the no-drug group did not differ significantly in their DBP responses between the 2 days. For SBP, the difference between the baby and no baby days was not significant for the cocaine group but was directionally consistent with the DBP difference, while for the no-drug group, there was a nonsignificant trend toward higher, not lower, baseline SBP and DBP on the baby contact day. Thus, this finding indicates that, contrary to a priori hypotheses, the manipulation of $10 \mathrm{~min}$ of warm mother-infant contact was associated with beneficial reductions in BP specifically in the cocaine-exposed mothers, an effect that persisted throughout the session during both high-and low-stress activities.

Including feeding method, race, education, and baby age as covariates in these models did not alter these results substantially, and in no case were these covariates significant. Furthermore, post hoc analyses separating the no-drug breast-feeding and no-drug bottlefeeding mothers into two subgroups failed to yield even trends toward differences in any laboratory BP measure between these two no-drug subgroups (all $P \mathrm{~s}>.20$ ). Other post hoc analyses restricting comparisons to the 10 cocaine-exposed mothers vs. the 11 no-drug bottle-feeding mothers yielded the same direction and approximate magnitude of differences on the no baby day for all significant group differences obtained in the initial analyses, although there was a loss of statistical power with the reduced sample size (baseline $110.3 / 74.0$ vs. $105.3 / 68.0, P<.15$; speech preparation $118.7 / 79.5$ vs. $110.2 / 71.5, P<.03$; replay $120.0 / 79.5$ vs. $109.2 / 72.9, P<.07$, respectively). As with the original analyses, no group differences in BP were significant on the baby day. Altogether, these post hoc analyses generated no support for the interpretation that differences in laboratory BP between the cocaine and no-drug groups were due to breast-feeding, or to group differences in baby age, mother's education, or race.

\subsection{Plasma oxytocin}

In contrast to laboratory BP differences, group differences in oxytocin were present on the baby contact day as well as the no baby day; the main effect of group was significant during the 2-day combined baseline and during speech on both days, $F \mathrm{~s}(1,34)=8.8,14.6$, and 23.1, $P$ s $<.0055, .0005$, and .0001 . As predicted, the cocaine-exposed mothers showed lower plasma oxytocin levels than the no-drug mothers at baseline $(1.1 \pm 0.2$ vs. $2.0 \pm 0.2 \mathrm{pg} / \mathrm{ml}, P$ $<$.0055). During the speech task on both the baby and no baby days, after adjustment for baseline differences, the cocaine group showed slight but significant decreases from their already low baseline levels $(-0.7 \pm 0.3$ and $-0.6 \pm 0.1 \mathrm{pg} / \mathrm{ml}$, respectively, $P<.025)$ while the no-drug mothers showed no reliable changes $(+0.3 \pm 0.2$ and $0 \pm 0.1 \mathrm{pg} / \mathrm{ml}$, respectively); thus, the responses to stress further exaggerated the oxytocin differences between the groups, $F \mathrm{~s}=7.8$ and $11.0, P \mathrm{~s}<.009$ and .002 ; see Fig. 3). 


\subsection{Behavior during baby contact in the laboratory}

Episodes of baby crying were rare among the no-drug group when the mother was playing with and holding the baby (mean $0.5 \pm 0.7$, difference from zero, $P=\mathrm{NS}$ ) but more frequent in the cocaine group (mean $2.4 \pm 1.0$, difference from zero, $P \leq .026$ ). Probably for this reason, mothers in the cocaine group spent more of the baby holding and play time on baby diaper checks, changing, burping, and other maintenance activities ( $8.3 \pm 2.0$ vs. $0.8 \pm 1.3, P$ $\leq .03)$. In terms of positive and negative maternal affective behaviors, mothers in the cocaine-exposed group laughed more often during baby play ( $4.4 \pm 0.9$ vs. $1.1 \pm 0.5, P \leq$. $003)$ and smiled nearly as often $(4.9 \pm 2.0$ vs. $7.5 \pm 1.3, P=\mathrm{NS})$ than those in the no-drug group. No group differences were obtained for mother frowning, kissing baby, eye contact, or for positive, neutral, or negative speech. Thus, overt signs of positive affect were as high or higher, and signs of negative affect were as low in the cocaine-exposed as in the no-drug mothers during baby play and holding.

\subsection{Twenty-four-hour BP and contact with baby at home}

For the 24-h period between the first and second laboratory sessions, all mothers continued to wear the ambulatory BP monitor and to collect all urine for norepinephrine and cortisol measures. Except during sleep, each time the monitor took a reading (four times per waking hour), the mother recorded her current activity and whether she had been holding or feeding her baby at any time since the last reading. The number of readings during and up to $1 \mathrm{~h}$ after feeding their babies did not differ reliably between the cocaine-exposed and no-drug groups after controlling for baby age ( $5.0 \pm 1.5$ vs. $6.7 \pm 0.9, P=\mathrm{NS})$. However, consistent with a priori hypotheses, there was a trend for mothers in the cocaine group to have fewer times that they held their babies when $n o t$ feeding them $(7.7 \pm 2.1$ vs. $11.2 \pm 1.2, P \leq .08$ one-tailed).

Lack of adequate sleep was considered as a potential confounder in these analyses. However, the cocaine and no-drug groups did not differ in hours of overnight sleep on the ambulatory monitoring day, and both groups achieved normal sleep durations ( $8.6 \pm 0.6$ vs. $7.9 \pm 0.4 \mathrm{~h}, P>.25$ ). In addition, duration of sleep was not correlated with baby age, or with depression, anxiety, or hostility scores (all $r \mathrm{~s} \leq+.10, P \mathrm{~s}=\mathrm{NS}$ ). Thus, no correction for differences in sleep duration was deemed necessary.

Mean SBP and DBP levels for sleep, postfeeding (including up to 60 min after nursing or bottle-feeding), and all nonfeeding waking activities depicted for the cocaine group (all bottle-feeders) and for the no-drug mothers subgrouped into breast-feeding and bottlefeeding mothers are shown in Figs. 4 and 5. Because feeding method was a significant covariate only for the postfeeding SBP measure, $F(1,30)=3.86, P<.05$, and postfeeding SBP was the only BP measure marginally different between the breast- and bottle-feeding subgroups of no-drug mothers, analyses for ambulatory SBP measures were performed two ways: (1) like all the other analyses in this study, comparing the cocaine group with the combined no-drug group, and (2) for postfeeding SBP only, comparing the cocaine group with the two no-drug subgroups divided by feeding method. In the two-group analysis, repeated-measures ANOVAs yielded a significant main effect for group across the three activity categories for both ambulatory SBP and DBP, $F(1,31)=9.05$ and $4.47, P \mathrm{~s} \leq .005$ and .043 , respectively, reflecting the consistently higher BP level of the cocaine group vs. the combined no-drug group. In the added three-group analysis for postfeeding SBP, the group effect remained significant, $F(2,30)=8.53, P<.0012$. Breast-feeding no-drug mothers had marginally lower postfeeding SBP levels than bottle-feeding no-drug mothers $(107.6 \pm 2.0$ vs. $113.7 \pm 2.3, P<.056)$ and both of these subgroups had lower postfeeding SBP levels than the cocaine group $(120.6 \pm 2.5, P \mathrm{~s}<.0003$ and .05 , respectively. 


\subsection{Norepinephrine, epinephrine and cortisol measures in laboratory and at home}

Groups did not differ in their plasma norepinephrine levels at baseline or during stress on either laboratory day, when all participants performed the same activities. However, in the home environment where individual differences in behavior and emotional reactions naturally occurred, the cocaine group had higher 24-h urinary norepinephrine levels than the no-drug group $[45.3 \pm 6.2$ vs. $29.9 \pm 3.7 \mathrm{mg} / 24 \mathrm{~h}, F(1,33)=4.48, P \leq .04]$. Urinary norepinephrine levels of no-drug breast- and bottle-feeders were not different ( $30.7 \pm 4.9$ vs. $28.9 \pm 5.7 \mathrm{mg} / 24 \mathrm{~h}, P>.80$ ), and the cocaine group differed from both, confirming that the enhanced norepinephrine activity at home was not due to feeding method.

Although their baseline plasma epinephrine levels were similar to the no-drug group (40.8 \pm 19.2 vs. $37.2 \pm 8.4 \mathrm{pg} / \mathrm{ml}, P=\mathrm{NS}$ ), the cocaine group showed a blunted epinephrine response to stress on both days after covarying for baseline levels. The no-drug group demonstrated epinephrine increases above baseline levels during stress on both the baby and no baby days $(+30.6$ and $+21.5 \mathrm{pg} / \mathrm{ml}, P<.026$ and .08$)$ while the cocaine group showed epinephrine decreases, not increases, during stress on both days $(-19.3$ and $-15.9 \mathrm{pg} / \mathrm{ml})$.

The cocaine group showed a trend towards lower serum cortisol levels at baseline than the no-drug group; however, this difference was specifically vs. the no-drug breast-feeders (3.89 \pm 1.5 vs. $6.37 \pm 1.5 \mathrm{pg} / \mathrm{ml}, P<.028)$, while they did not differ from the no-drug bottlefeeders $(4.68 \pm 1.8, P=\mathrm{NS})$. In contrast, their 24-h urinary cortisol levels were lower than the no-drug bottle-feeding subgroup $(49.0 \pm 15.0$ vs. $76.5 \pm 18.0 \mathrm{mg} / 24 \mathrm{~h}, P \leq .027)$, while not differing from the breast-feeding mothers $(52.0 \pm 15.9, P=\mathrm{NS})$. Together, these findings suggest that cortisol may be modestly altered in this group, but might best be described as low-normal.

\subsection{Negative affect, social support, stress, and mastery measures}

As depicted in Table 2, the cocaine group reported higher levels of negative affect and recent stress along with lower levels of social support and control over their lives compared to the no-drug group. Most notably, the cocaine mothers scored higher than no-drug mothers in both total hostility (sum of the 15 items in the hostile affect, aggressive responding, and cynical hostility Ho subscales) and in depressive symptoms on the BDI, $F \mathrm{~s}(1,34)=4.01$ and $7.58, P \mathrm{~s} \leq .05$ and .01 , respectively. The cocaine group scored lower on the Pearlin Mastery Scale and on the ISEL total social support score, $F \mathrm{~s}=10.58$ and 4.61, $P \mathrm{~s} \leq .003$ and .04 , respectively. They also tended to report more life stress on the Perceived Stress Scale (reflecting perception of total stress exposure and sense of being overwhelmed during the past 30 days) and higher state anxiety on the ambulatory monitoring day, $F \mathrm{~s}=3.01$ and $3.12, P \leq .09$. No significant group differences were seen in terms of the Anger Expression Scale, the Daily Stress Inventory (hassles and stressors reported for the 24-h period of home monitoring), or Trait Anxiety. Altogether, this profile suggests a pattern of more aggressive responding and negative mood combined with higher stress exposure in recent weeks and less stress buffering from feeling in control over their lives or from emotional and tangible support provided by family and friends.

\subsection{Interrelationships between oxytocin and other outcome measures}

Based on directional hypotheses, we also examined simple correlations between oxytocin levels and other outcome measures, specifically BP measures and measures of stress and stress buffers, in the sample as a whole $(N=35$; see Table 3$)$. Oxytocin level at baseline was unrelated to any BP measure, but higher oxytocin during speech stress on the baby contact day was related to lower ambulatory SBP after baby feeding, to lower laboratory SBP at speech preparation on the baby contact day (the period immediately after baby contact), and to lower laboratory DBP at baseline on the baby contact day $(r=-0.32,-0.29$, and -0.30 , 
respectively, $P<.05)$. Oxytocin levels at baseline and during speech were both positively correlated with higher Pearlin Mastery Scale scores $(r=+0.39$ and +0.33$)$ and with greater total social support scores on the ISEL $(r=+0.29$ and +0.40 , respectively, $P<.05)$.

Relationships with other affective and stress measures were in the predicted directions but nonsignificant (see Table 3).

In contrast to oxytocin, lower serum cortisol was associated only with increased state anxiety $(r=-0.42, P<.029)$, but not to other psychological measures $(P \mathrm{~s} \geq .20)$. Higher plasma norepinephrine at baseline and during stress was linked to higher depression scores $(r \mathrm{~s}=+0.31$ and $+0.35, P \mathrm{~s}<.05)$ but not to other measures $(P \mathrm{~s} \geq .20)$. Urinary cortisol and norepinephrine both showed no reliable associations with any of these indexes of psychological distress $\left(P_{\mathrm{s}} \geq .13\right)$.

\section{Discussion}

A consistent pattern of parallels was observed between the responses of mothers in this study who used cocaine during their pregnancy and animal models of gestational cocaine exposure. In animal models, gestational cocaine exposure leads to deficits in oxytocinergic activity, and to diminished maternal but increased aggressive behaviors, effects similar to those seen in dams treated centrally with an oxytocin antagonist rather than cocaine (Johns, Lubin et al., 1997; Johns, Noonan et al., 1997, Johns et al., 1998; Lubin, Elliott et al., 2003). In the present investigation, mothers with gestational cocaine exposure reported more hostility and depressed mood, a trend towards more life stress over the past month, and less social support and sense of mastery and control in their lives. These mothers tended to hold their babies less frequently over a 24-h period in their home environments, a day when they showed greater production of the stress hormone, norepinephrine, and higher BP across all activities, both asleep and awake, compared with other mothers of infants. Consistent with a priori hypotheses, all of these differences were linked in the cocaine group with lower plasma levels of oxytocin and diminished oxytocin responses to mental stress in the laboratory. In the total sample of 35 mothers studied, higher oxytocin levels at baseline and during stress were correlated with greater social support from others and with greater sense of control over events in their lives.

The central oxytocinergic system influences many areas of the brain involved in both emotion and cardiovascular control, in addition to its own actions on oxytocin receptors in the periphery that can directly modulate $\mathrm{BP}$ and other cardiovascular measures. Recent studies in oxytocin knockout mice have confirmed that these animals have abnormal autonomic and baroreflex control over heart rate (HR) and BP, and that female mice lacking oxytocin have increased anxiety-like behaviors (Mantella, Vollmer, Li, \& Amico, 2003; Michelini, Marcelo, Amico, \& Morris, 2003). Other recent research in both male and female animal models indicates that in addition to its production within the hypothalamus, oxytocin is produced and released in the heart and the vasculature, and that oxytocin specifically activates receptors that are present in both sites, leading to decreases in HR, contractile force and vascular resistance (Jankowski et al., 2000; Mukaddam-Daher, Yin, Roy, Gutkowska, \& Cardinal, 2001; Thibonnier et al., 1999). Thus, oxytocin may act both centrally and peripherally to lower BP. Although the present study is correlational in nature, and no causeand-effect conclusions can be drawn, the direction of the oxytocin and BP effects are consistent with the possible contribution of low oxytocinergic activity to the increased BP in the cocaine-exposed mothers.

In addition to their deficient oxytocin activity, the cocaine-exposed mothers demonstrated other alterations in neuromodulating systems: excessive 24-h urinary norepinephrine levels combined with low-normal serum and urinary cortisol levels. The heightened 
norepinephrine levels may be linked to increased stress exposure and decreased coping resources, as suggested by their psychosocial ratings, but increased central adrenergic activity can also directly contribute to both increased BP and negative affective states, such as anxiety, depression, and hostility, all of which were seen in this group. Depressive symptoms have also been correlated with suppressed baseline cortisol but enhanced cortisol and ACTH increases during cocaine administration to dependent individuals, suggesting that for some individuals, depressive symptoms linked to altered HPA axis activity may precede and be temporarily alleviated by cocaine use (Elman et al., 1999). Although it is not possible in the present study to determine whether the greater depressive and anxiety symptoms in the cocaine-exposed mothers preceded and enhanced vulnerability to cocaine abuse, prior research by Abraham and Fava (1999) used blinded retrospective histories to examine whether substance abuse or depression came first in patients with a history of both. They found that patients with a history of cocaine abuse had more lifetime episodes of depression (mean 12.2 episodes) compared to patients who had abused other drugs, particularly opiates (mean 3.7 episodes), and that the depressive episodes began a number of years before their cocaine use. These observations are consistent with the theory that cocaine use may in some cases begin as part of an effort at self-medication for depressive and anxiety symptoms. Our study's findings of milder psychological symptoms that were linked to blunted cortisol but increased norepinephrine levels suggest that such residual markers may persist even during abstinence and recovery. It must be emphasized that, in the present study, any mother currently using antidepressants or meeting diagnostic criteria for clinical depression was excluded.

There are a number of limitations to this investigation and the findings must therefore be generalized only with caution. First and most important, the subgroup with cocaine exposure during pregnancy was small and highly selected; all of them were enrolled in a rehabilitation program while pregnant, and had full-term, uncomplicated deliveries, and all were not currently depressed or on antidepressants. Thus, our sample may be representative of only the less affected part of this population. Haller, Miles, and Dawson (2003) compared pregnant substance abusers who enrolled in a similar program $(n=102)$ vs. the minority who declined such treatment $(n=23)$ in a sample much like ours from the southeastern United States where the majority were unemployed African Americans with no college education. They found no group differences in these demographic variables, but reported that women who enrolled in treatment had greater drug severity, emotional distress, depression, and other psychological symptoms than those who did not, a profile similar to the cocaine-exposed participants in our study. Nevertheless, these women did make up approximately $80 \%$ of the pregnant substance abusers they screened, suggesting that our findings may apply to the majority of women in this group. Second, the present study is essentially correlational in nature and no definitive evidence can be provided that oxytocin deficits are a major or even a minor contributing cause of the higher BP or greater hostility, anxiety, and depressive symptoms in the cocaine-exposed group. BP differences between the two groups might plausibly be due to many other variables, such as direct and indirect residual effects of that period of prior drug use (including poorer diet, sleep, and exercise patterns) and long-term environmental stress exposure. All mothers in the cocaine-exposed group, however, had no recent exposure to drugs as confirmed by their statements and by urine screens required by their rehabilitation program, so that direct effects of cocaine on BP are unlikely to be the source of our group difference. The majority of recent investigations have indicated that although cocaine can increase BP and circulating catecholamines transiently in a dose-dependent fashion, these elevations normalize within $24 \mathrm{~h}$ after most recent use, after which age-adjusted BP levels of cocaine users do not differ from those of nonusers (Brecklin \& Bauman, 1999; Brecklin et al., 1998; Foltin, Ward, Haney, Hart, \& Collins, 2003; Sofuoglu, Nelson, Babb, \& Hatsukami, 2001). Stress exposure and lifestyle differences provide potentially important contributions to our findings. Although they did 
not report greater exposure to stressors on the day of ambulatory monitoring, mothers in the cocaine-exposed group did report less control over the stressful events they experienced in the past month (based on the Perceived Stress and Mastery Scales) and less access to social support to buffer against the effects of high stress. According to Seeman, Singer, Ryff, Dienberg-Love, and Levy-Sorms (2002); this combination is one that frequently leads to greater total allostatic load with significant implications for higher BP and depressive symptoms, and other health outcomes. Thus, within the present design, it is not possible to separate these factors from oxytocin's influence on the BP and affect differences observed.

The range of infant ages within this sample, from age 1 month to 11 months, represents a period of dramatic changes in behavioral development for the infant, in experience with parenting skills for the mother, and in attachment and bonding for the mother-infant dyad. A more restricted age range would have been preferable to reduce error variance; however, both the cocaine and the no-drug groups included infants near the upper and lower ends of this age range, and adjustment for infant age in no case affected our group differences. This wide age range was a factor in our decision to limit our infant behavior and mother-infant interaction assessments to very few measures, although such dyadic interactions deserve greater attention in future, larger scale studies. Clearly, it would also have been better to have had both groups matched for feeding method, particularly because the act of breastfeeding typically involves a surge in oxytocin activity. The fact that all of the cocaine group elected to bottle-feed rather than breast-feed was in fact a sign of their compliance with medical advice, which was directed at protection of the infant, whereas in the no-drug group, most women who chose bottle-feeding did so against the weight of medical advice. As both primary and secondary analyses demonstrated, feeding method, education, and racial group differences did not account for the overall pattern of group differences obtained. Even in the case of the exception to this overall pattern, postfeeding SBP, the cocaine group maintained higher BP levels than either the bottle- or breast-feeding no-drug mothers.

Although the pattern of differences in the cocaine-exposed mothers is consistently in the direction suggesting increased stress and heightened sympathetic nervous system activity, more negative affect and less warm baby contact, the laboratory data on BP offered some more positive signs. That is, on the day when all mothers held and played with their babies for $10 \mathrm{~min}$ prior to stress exposure in the laboratory, the cocaine-exposed group no longer had higher BP than the comparison group of mothers. In fact, their BP levels were significantly reduced relative to their own levels on the day when they had no baby contact time. This observation suggests that although their oxytocinergic pathways may be less responsive than other mothers, the cocaine-exposed mothers appear to benefit when encouraged to spend warm contact time with their infants. Given the pharmacological properties of cocaine, such contact may be acting through other mediators that we did not assess directly, such as endogenous opioids, serotonergic systems, or via altered autonomic balance. A recent animal publication does indicate that some alterations in the serotonergic and dopaminergic systems are seen in specific brain regions at the time when aggression is increased by gestational cocaine treatment, although the relevance of such changes are presently unknown (Lubin, Cannon, Black, Brown, \& Johns, 2003). Alternatively, they may demonstrate an increase in oxytocin activity that was not detected given the short half life of oxytocin in plasma and the timing of our blood sampling. It was also notable that in the laboratory, when instructed to hold and play with their babies, the cocaine-exposed mothers showed equal or greater positive affective behaviors and no excessive negative affective behaviors compared to the no-drug mothers, although their babies cried more often; greater crying and irritability in infants prenatally exposed to cocaine has been reported as typical (Keller \& Snyder-Keller, 2000). This positive affect when interacting with their infants in the laboratory is in contrast to the questionnaire data describing the mothers' typical affective states, in which the cocaine group reported greater hostility and depressed mood. It 
is possible that the baby serves as a security or transitional object to these mothers, a signal of the positive changes that they have begun to make in their lives. In addition, the significance of warm touch with their infants may be especially powerful for this group of mothers, especially if they rarely receive warm touch contact or emotional support from others, as suggested by their low social-support scores. One possible interpretation is that if the cocaine-exposed mothers could have more warm physical contact time with their infants at home, they might benefit by improving their own mood and their BP levels.

Although the mothers did show benefit from baby contact in the laboratory setting, their diaries indicated that the cocaine-exposed mothers held and played with their babies somewhat less frequently at home than the other mothers. This may explain why the ambulatory home BP differences between the groups were more similar to the BP differences observed in the laboratory on the day of no baby contact. It might have been interesting to assess whether the mothers would have interacted with their babies in the laboratory if given a choice rather than following the study protocol. These observations suggest that the mothers may need encouragement and even direct training and practice in holding, touching, and making warm contact with their babies. Extended physical contact between mother and infant has been shown previously to have significant health- and growth-enhancing benefits for the infant, especially if premature or with other health problems at birth (Chwo et al., 2002). Some of these studies have also demonstrated that extended mother-child contact has benefits for the mother and father as well, decreasing negative affect and perceived stress, and increasing sensitive maternal/paternal behavior (Feldman, Eidelman, Sirota, \& Weller, 2002).

Reports of poor parenting patterns and increased antisocial and physically aggressive behaviors among current and former cocaine users have encouraged further study aimed at focusing on factors involved in mediating these behaviors and on designing interventions to replace such behaviors with more positive ones. Recent work by Hien and Miele (2003) documented excessive use of emotion-focused coping and pointed to the importance of emotion regulation in the prevention of violence in drug-abusing women. Coyer (2003) interviewed women recovering from cocaine addiction about parenting, and reported that major themes included inability to control impatience and anger as well as the mother's own exposure as a child to poor or absent parenting (based on abandonment and/or a dysfunctional family). Animal studies have shown a critical link between early life experience, later maternal behavior, and oxytocin. Mother rats that have higher rates of maternal behavior (licking and grooming their pups) than other dams also have markedly higher oxytocin receptor density in the amygdala (Champagne \& Meaney, 2001).

Significantly lower oxytocin levels are found in the amygdala during the postpartum period when rat mothers gestationally treated with cocaine are compulsively aggressive and aggression is significantly elevated in nontreated rat mothers when amygdaloid oxytocin receptors are blocked during the postpartum period (Johns et al., 1998; Lubin, Elliott et al., 2003). Increases in oxytocin levels in brain regions of the postpartum rat, such as the medial preoptic area of the hypothalamus, ventral tegmental region, and amygdala, are associated with better maternal behavior and, for amygdala only, decreased aggression (Elliott et al., 2001; Johns et al., 1998). These data suggest that increasing oxytocin levels and binding during the postpartum period by some means (perhaps contact) may enhance different aspects of maternal behavior. Interestingly, high rates of maternal behavior can be also be enhanced by selective breeding, suggesting a genetic basis, but cross-fostering and other interventions have confirmed that when the female pups mature, they will demonstrate maternal behaviors that replicate their early experience (prenatal and rearing), not their genetic background. This transgenerational effect makes it doubly important that efforts be made to encourage former cocaine-exposed mothers to increase (and model) good parenting behaviors (Champagne \& Meaney, 2001; Johns et al., 2002). Stronger bonding with their 
own children as well as enhanced psychoemotional state are both important components to address in future, larger scale research (particularly intervention studies) involving this population. This and prior investigations indicate a role for warm physical contact and oxytocin within such research, for which the present study represents only an encouraging first step.

\section{Acknowledgments}

This research was supported in part by NIH grants HL64927 to KCL, HD/DK37268 to JAA, DA/AA13362 awarded by the Federal Neglect Consortium to JMJ, and GCRC RR00046 to UNC. We gratefully acknowledge the invaluable technical assistance of Sunny H. Chung, Monica Adamian, Tara E. Smith, Hou-ming Cai and the secretarial assistance of Dot Faulkner.

\section{References}

Abraham HD, Fava M. Order of onset of substance abuse and depression in a sample of depressed outpatients. Comprehensive Psychiatry. 1999; 40:44-50. [PubMed: 9924877]

Altemus M, Redwine LS, Leong YM, Frye CA, Porges SW, Carter CS. Responses to laboratory psychosocial stress in postpartum women. Psychosomatic Medicine. 2001; 63:814-821. [PubMed: 11573030]

Amico JA, Ervin MG, Leake RD, Fisher DA, Finn FM, Robinson AG. A novel oxytocin-like and vasotocin-like peptide in human plasma after administration of estrogen. Journal of Clinical Endocrinology and Metabolism. 1985; 60:5-12.

Amico JA, Johnston JM, Vagnucci AH. Suckling induced attenuation of plasma cortisol in postpartum lactating women. Endocrinology Research. 1994; 20:79-87.

Barefoot JC, Dodge KA, Peterson BL, Dahlstrom WG, Williams RB. The Cook Medley Hostility Scale: Item content and ability to predict survival. Psychosomatic Medicine. 1989; 51:46-57. [PubMed: 2928460]

Barefoot JC, Larsen S, von der Lieth L, Schroll M. Hostility, incidence of acute myocardial infarction, and mortality in a sample of older Danish men and women. American Journal of Epidemiology. 1995; 142:477-484. [PubMed: 7677126]

Bauman PS, Dougherty FE. Drug addicted mothers parenting and their children's development. The International Journal of the Addictions. 1983; 18:291-302. [PubMed: 6874152]

Bays J. Substance abuse and child abuse: Impact of addiction on the child. Pediatric Clinics of North America. 1990; 37:881-904. [PubMed: 2199920]

Beck AT, Ward CH, Mendelson M, Mock J, Erbaugh J. An inventory for measuring depression. Archives of General Psychiatry. 1961; 4:561-571. [PubMed: 13688369]

Beckham JC, Calhoun PS, Glenn DM, Barefoot JC. Post traumatic stress disorder, hostility and health in women: A review of current research. Annals of Behavioral Medicine. 2002; 24:219-228. [PubMed: 12173679]

Brantley PJ, Waggoner CD, Jones GN, Rappaport NB. A daily stress inventory: Development, reliability and validity. Journal of Behavioral Medicine. 1987; 10:61-74. [PubMed: 3586002]

Brecklin CS, Bauman JL. Cardiovascular effects of cocaine: Focus on hypertension. Journal of Clinical Hypertension. 1999; 1:212-217. [PubMed: 11416615]

Brecklin CS, Gopaniuk-Folga A, Kravetz T, Sabah S, Singh A, Arruda JA, Dunea G. Prevalence of hypertension in chronic cocaine users. American Journal of Hypertension. 1998; 11:1279-1283. [PubMed: 9832169]

Brookings JB, Bolton B. Confirmatory factor analysis of the Interpersonal Support Evaluation List. American Journal of Community Psychology. 1988; 16:137-147. [PubMed: 3369379]

Burns K, Chethik L, Burns WJ, Clark R. Dyadic disturbances in cocaine-abusing mothers and their infants. Journal of Clinical Psychology. 1991; 313:666-669.

Champagne F, Meaney MJ. Like mother, like daughter: Evidence for non-genomic transmission of parental behavior and stress responsivity. Progress in Brain Research. 2001; 133:287-302.

[PubMed: 11589138] 
Chwo MJ, Anderson GC, Good M, Dowling DA, Shiau SH, Chu DM. A randomized controlled trial of early kangaroo care for preterm infants: Effects on temperature, weight, behavior and acuity. Journal of Nursing Research. 2002; 10:129-142. [PubMed: 12119598]

Cohen, S. Measuring the functional components of social support. In: Sarason, I.; Sarason, B., editors. Social support: Theory, research and applications. The Hague, Netherlands: Martinus Nijhoff; 1985. p. 73-94.

Cohen S, Kamarck T, Mermelstein R. A global measure of perceived stress. Journal of Health and Social Behavior. 1983; 24:385-396. [PubMed: 6668417]

Cook WW, Medley DM. Proposed hostility and pharisaic-virtue for the MMPI. Journal of Applied Psychology. 1954; 328:414-418.

Coyer SM. Women in recovery discuss parenting while addicted to cocaine. American Journal of Maternal Child Nursing. 2003; 28:45-49. [PubMed: 12514356]

Elliott J, Lubin DA, Johns JM. Acute cocaine alters oxytocin levels in the medial preoptic area and amygdala of lactating rat dams: Implications for cocaine-induced changes in maternal behavior and maternal aggression. Neuropeptides. 2001; 35:1-8. [PubMed: 11346306]

Elman I, Breiter HC, Gollub RL, Krause S, Kantor HL, Baumgartner WA, Gastfriend DR, Rosen BR. Depressive symptomatology and cocaine-induced pituitary-adrenal axis activation in individuals with cocaine dependence. Drug and Alcohol Dependence. 1999; 56:39-45. [PubMed: 10462091]

Feldman R, Eidelman AI, Sirota L, Weller A. Comparison of skin-to-skin (kangaroo) and traditional care: Parenting outcomes and preterm infant development. Pediatrics. 2002; 119:16-26. [PubMed: 12093942]

Fineman NR, Beckwith L, Howard H, Espinosa M. Maternal ego development and mother-infant interaction in drug-abusing women. Journal of Substance Abuse Treatment. 1997; 14:307-317. [PubMed: 9368207]

Foltin RW, Ward AS, Haney M, Hart CL, Collins ED. The effects of escalating doses of smoked cocaine in humans. Drug and Alcohol Dependence. 2003; 70:149-157. [PubMed: 12732408]

Girdler SS, Sherwood A, Hinderliter AL, Leserman J, Costello NL, Straneva PA, Pedersen CA, Light KC. Biologic correlates of abuse in women with premenstrual dysphoric disorder and healthy controls. Psychosomatic Medicine. 2003; 65:849-856. [PubMed: 14508031]

Goldenberg RL, Hickey CA, Cliver SP, Gotlieb S, Woolley TW, Hoffman HJ. Abbreviated scale for the assessment of psychosocial status in pregnancy: Development and evaluation. Acta Obstetricia et Gynecologica Scandinavica. 1997; 165(Suppl 1):19-29.

Goldman-Fraser, J. Unpublished dissertation. University of North Carolina; 1997. Cognitive influences on mother-infant interaction: A focus on substance-dependent women in treatment during pregnancy and postpartum.

Haller DL, Miles DR, Dawson KS. Factors influencing treatment enrollment by pregnant substance abusers. American Journal of Drug and Alcohol Abuse. 2003; 29:117-131. [PubMed: 12731684]

Heck H, de Castro JM. The caloric demand of lactation does not alter spontaneous meal patterns, nutrient intakes, or moods of women. Physiology and Behavior. 1993; 54:641-648. [PubMed: 8248340]

Hien DA, Miele GM. Emotion-focused coping as a mediator of maternal cocaine abuse and antisocial behavior. Psychology of Addictive Behaviors. 2003; 17:49-55. [PubMed: 12665081]

Holst S, Uvnas-Moberg K, Petersson M. Postnatal oxytocin treatment and postnatal stroking of rats reduce blood pressure in adulthood. Autonomic Neuroscience. 2002; 99:85-90. [PubMed: 12241092]

Howard J, Beckwith L, Espinosa M, Tyler R. Development of infants born to cocaine-abusing women: Biologic/maternal influences. Neurotoxicology and Teratology. 1995; 17:403-411. [PubMed: 7565486]

Jankowski M, Wang D, Hajjar F, Mukaddam-Daher S, McCann S, Gutkowska J. Oxytocin and its receptors are synthesized in the rat vasculature. Proceedings of the National Academy of Sciences of the United States of America. 2000; 97:6207-6211. [PubMed: 10811917]

Johns JM, Lubin DA, Black M, Elliott JC, Joyner PW, Lomas LM, Middleton CL, Holfler V, Knupp $\mathrm{K}$, Goldhammer R. Intergenerational effects of cocaine on rodent maternal behavior: Preliminary results. Abstracts-Society for Neuroscience (\#878.17). 2002 
Johns JM, Lubin DA, Walker CH, Meter KE, Mason GA. Chronic gestational cocaine treatment decreases oxytocin levels in the medial preoptic area, ventral tegmental area and hippocampus in Sprague-Dawley rats. Neuropeptides. 1997; 31:439-443. [PubMed: 9413020]

Johns JM, Nelson CJ, Meter KE, Lubin DA, Couch CD, Ayers A, Walker CH. Dose-dependent effects of multiple acute cocaine injections on maternal behavior and aggression in Sprague-Dawley rats. Developmental Neuroscience. 1998; 20:525-532. [PubMed: 9858841]

Johns JM, Noonan LR, Zimmerman LI, Li L, Pedersen CA. Effects of chronic and acute cocaine treatment on maternal behavior and aggression. Behavioral Neuroscience. 1994; 108:107-112. [PubMed: 8192835]

Johns JM, Noonan LR, Zimmerman LI, Li L, Pedersen CA. Effects of short and long term withdrawal from gestational cocaine treatment on maternal behavior and aggression in Sprague-Dawley rats. Developmental Neuroscience. 1997; 19:368-374. [PubMed: 9215883]

Johnson HL, Rosen T. Mother-infant interaction in a multirisk population. American Journal of Orthopsychiatry. 1990; 60:281-288. [PubMed: 2098001]

Keller RW Jr, Snyder-Keller A. Prenatal cocaine exposure. Annals of the New York Academy of Sciences. 2000; 909:217-232. [PubMed: 10911932]

Kelly SJ, Walsh JH, Thompson K. Birth outcomes, health problems, and neglect with prenatal exposure to cocaine. Pediatric Nursing. 1992; 17:130-136.

Keverne, E.; Kendrick, K. Oxytocin facilitation of maternal behavior in sheep. In: Pedersen, CA.; Caldwell, JD.; Jirikowski, GF.; Insel, TR., editors. Oxytocin in maternal, sexual, and social behaviors, Annals of the New York Academy of Sciences. Vol. 652. 1992. p. 83-101.

Knox SS, Uvnas-Moberg K. Social isolation and cardiovascular disease: An atherosclerotic pathway? Psychoneuroendocrinology. 1998; 23:877-890. [PubMed: 9924742]

Koo-Loeb JH, Costello N, Light KC, Girdler SS. Women with eating disorder tendencies display altered cardiovascular, neuroendocrine, and psychosocial profiles. Psychosomatic Medicine. 2000; 62:539-548. [PubMed: 10949100]

Lawson MS, Wilson GS. Parenting among women addicted to narcotics. Child Welfare. 1980; 59:6779. [PubMed: 7358006]

Light KC, Smith TE, Johns JM, Hofheimer JA, Amico JA. Oxytocin responsivity in mothers of infants: An initial study of relationships to laboratory and home blood pressure, affect and perceived support. Health Psychology. 2001; 19:560-567. [PubMed: 11129359]

Lubin DA, Cannon JB, Black MC, Brown LE, Johns JM. Effects of chronic cocaine on monoamine levels in discrete brain structures of lactating rat dams. Pharmacology, Biochemistry and Behavior. 2003; 74:449-454.

Lubin DA, Elliott JC, Black MC, Johns JM. An oxytocin antagonist infused into the central nucleus of the amygdala increases maternal aggressive behavior. Behavioral Neuroscience. 2003; 117:195201. [PubMed: 12708515]

Mantella RC, Vollmer RR, Li X, Amico JA. Female oxytocin-deficient mice display enhanced anxiety-related behavior. Endocrinology. 2003; 144:2291-2296. [PubMed: 12746288]

Martin, P.; Bateson, P. Measuring behavior: An introductory guide. 2. New York: Cambridge University Press; 1993.

Mezzacappa ES, Katkin E. Breast-feeding is associated with reduced perceived stress and negative mood in mothers. Health Psychology. 2002; 21:187-193. [PubMed: 11950109]

Michelini LC, Marcelo MC, Amico J, Morris M. Oxytocinergic regulation of cardiovascular function: Studies in oxytocin-deficient mice. American Journal of Physiology. Heart and Circulatory Physiology. 2003; 284:H2269-H2276. [PubMed: 12531722]

Modahl, C.; Newton, N. Mood state difference between breast and bottle-feeding mothers. In: Carenza, L.; Zinchella, L., editors. Emotion and Reproductions: Proceedings of the Serrano Symposium. Vol. 20B. New York: Academic Press; 1979. p. 819-822.

Mukaddam-Daher S, Yin Y, Roy J, Gutkowska J, Cardinal R. Negative inotropic and chronotropic effects of oxytocin. Hypertension. 2001; 38:292-296. [PubMed: 11509492]

Murphy JM, Jellinik M, Quinn D, Smith G, Poitrast FG, Goshko M. Substance abuse and serious child mistreatment; prevalence, risk and outcome in a court sample. Child Abuse and Neglect. 1991; 15:197-211. [PubMed: 2043972] 
Nelson CJ, Meter KE, Walker CH, Ayers AA, Johns JM. A dose-response study of chronic cocaine on maternal behavior in rats. Neurotoxicology and Teratology. 1998; 20:657-660. [PubMed: 9831128]

Pedersen CA, Caldwell JD, Walker CH, Ayers G, Mason GA. Oxytocin activates the postpartum onset of rat maternal behavior in the ventral tegmental and medial peoptic areas. Behavioral Neuroscience. 1994; 108:107-112. [PubMed: 8192835]

Petersson M, Alster P, Lundeberg T, Uvnas-Moberg K. Oxytocin causes a long-term decrease of blood pressure in female and male rats. Physiology and Behavior. 1996; 60:1311-1315. [PubMed: 8916187]

Petersson M, Uvnas-Moberg K, Erhardt S, Engberg G. Oxytocin increases locus coeruleus alpha 2 adrenoceptor responsiveness. Neuroscience Letters. 1998; 255:115-118. [PubMed: 9835228]

Robinson DA, Wei F, Wang GD, Li P, Kim SJ, Vogt SK, Muglia LJ, Zhuo M. Oxytocin mediates stress-induced analgesia in adult mice. Journal of Physiology. 2002; 540:593-606. [PubMed: 11956346]

Seeman TE, Singer BH, Ryff CD, Dienberg-Love G, Levy-Sorms L. Social relationships, gender, and allostatic load across two age cohorts. Psychosomatic Medicine. 2002; 64:395-406. [PubMed: 12021414]

Skaff MM, Pearlin LI, Mullan JT. Transitions in caregiving career; effects on sense of mastery. Psychology and Aging. 1996; 11:247-257. [PubMed: 8795053]

Sofuoglu M, Nelson D, Babb DA, Hatsukami DK. Intravenous cocaine increases plasma epinephrine and norepinephrine in humans. Pharmacology, Biochemistry and Behavior. 2001; 68:455-459.

Spielberger, C. Manual for the state-trait anxiety inventory; STAI (Form Y). Palo Alto, CA: Consulting Psychologists Press; 1983.

Spielberger, C.; Syderman, S. State-trait anxiety inventory and state-trait anger expression inventory. In: Maruish, M., editor. The use of psychological tests for treatment, planning and outcome assessment. Hillsdale, NJ: LEA; 1994. p. 291-321.

Thibonnier M, Conarty DM, Preston JA, Plenischer CL, Dweik RA, Erzurum SC. Human vascular endothelial cells express oxytocin receptors. Endocrinology. 1999; 140:1301-1309. [PubMed: 10067857]

Uvnas-Moberg K, Widstrom A-M, Nessen E, Bjorvell H. Personality traits in women 4 days postpartum and their correlation with plasma levels of oxytocin and prolactin. Journal of Psychosomatic Obstetrics and Gynaecology. 1990; 11:261-273.

Virden SF. The relationship between infant feeding and maternal role adjustment. Journal of NurseMidwifery. 1988; 33:31-35. [PubMed: 3339468]

Williams-Petersen M, Myers BJ, Degen HM, Knisely JS, Elswick RK, Schnoll SS. Drug-using and nonusing women: Potential for child abuse, child rearing attitudes, social support, and affection for expected baby. The International Journal of the Addictions. 1994; 29:1631-1643. [PubMed: 7836024] 


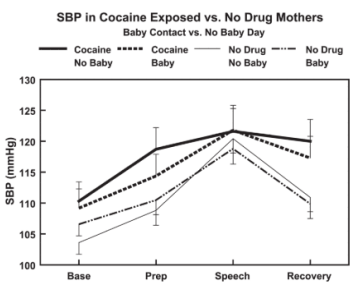

Fig. 1.

Differences in laboratory systolic blood pressure (SBP) levels during baseline, speech preparation, active speech, and recovery periods on the baby contact vs. the no baby test days for the cocaine-exposed vs. no-drug groups. Cocaine group SBP>no-drug group SBP for baseline, preparation, and recovery on the no baby day, $P \mathrm{~s}<.075, .034$, and .039 , respectively, but during speech on both days and during all events on the baby day, all $P$ s $=$ NS; effect sizes for group differences on the no baby vs. baby days are 0.10 vs. 0.02 for baseline, 0.14 vs. 0.02 for preparation, 0 vs. 0.01 for speech, and 0.14 vs. 0.08 for recovery. 


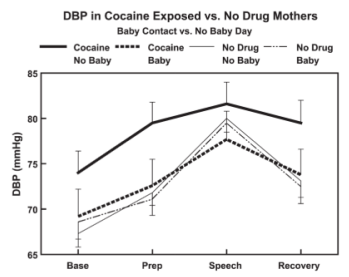

Fig. 2.

Differences in laboratory diastolic blood pressure (DBP) levels during baseline, speech preparation, active speech, and recovery periods on the baby contact vs. the no baby test days for the cocaine-exposed vs. no-drug groups. Cocaine group DBP>no-drug group DBP for baseline, preparation, and recovery on the no baby day, $P$ s $<.026, .007$, and .066, respectively, but during speech on both days and during all events on the baby day, all $P \mathrm{~s}=$ NS; effect sizes for group differences on the no baby vs. baby days are 0.16 vs. 0 for baseline, 0.24 vs. 0.01 for preparation, 0 vs. 0.01 for speech, and 0.14 vs. 0 for recovery. In addition, for the cocaine group only, DBP levels on the no baby day are higher than on the baby day, $P<.05$ for preparation and recovery, $P<.07$ for baseline and speech. 


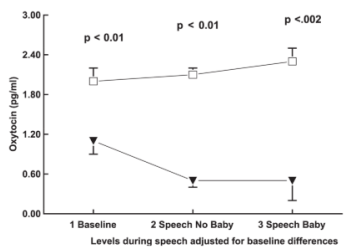

Fig. 3.

Plasma oxytocin levels during single combined baseline and during speech on both the baby contact and no baby test days are lower for the cocaine-exposed vs. no-drug groups. 


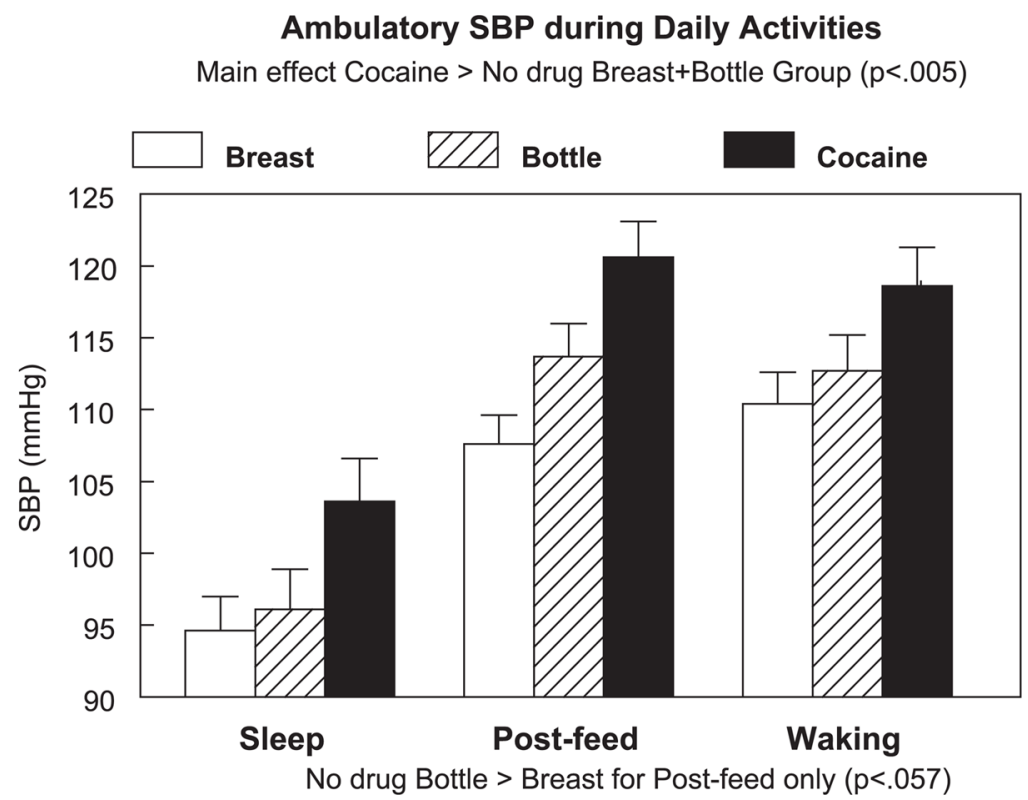

Fig. 4.

Mean ambulatory levels of SBP during sleep, postfeeding, and all other waking activities are higher for the cocaine vs. the no-drug group (breast-feeding + bottle-feeding mothers combined), $P<.005$. 
Ambulatory DBP during Daily Activities

Main Effect Cocaine > No drug Breast+Bottle Group $(p<.05)$

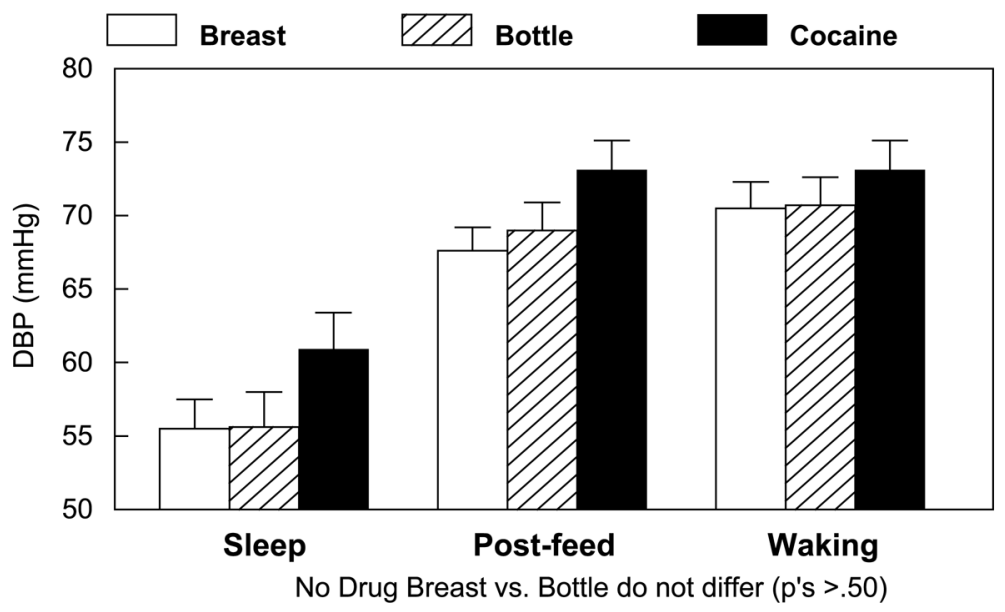

Fig. 5.

Mean ambulatory levels of DBP during sleep, postfeeding, and all other waking activities are higher for the cocaine vs. the no-drug group (breast-feeding + bottle-feeding mothers combined), $P<.05$. 
Table 1

Demographic information on cocaine vs. no-drug groups

\begin{tabular}{|c|c|c|c|}
\hline Variable & Cocaine & No drug & Difference $P$ \\
\hline$n$ & 10 & 25 & \\
\hline Mother age (years) & $30.2 \pm 1.5$ & $29.0 \pm 1.1$ & NS \\
\hline Baby age (months) ${ }^{a}$ & $3.5 \pm 1.2$ & $5.5 \pm 0.5$ & $P<.10$ \\
\hline Range & $1-11$ & $2-11$ & \\
\hline Ambulatory sleep (h) & $8.6 \pm 0.6$ & $7.9 \pm 0.4$ & NS \\
\hline Body mass index $\left(\mathrm{kg} / \mathrm{m}^{2}\right)$ & $27.5 \pm 1.6$ & $25.0 \pm 1.1$ & NS \\
\hline Alcohol beverage/day & $0.3 \pm 0.1$ & $0.4 \pm 0.1$ & NS \\
\hline Range & $0-1$ & $0-1$ & \\
\hline Breast-feed/bottle-feed ${ }^{a}$ & $0: 10$ & $14: 11$ & $P<.001$ \\
\hline Black/White $^{a}$ & $8: 2$ & $7: 18$ & $P<.005$ \\
\hline Percentage with no current spouse/partner & 40 & 25 & NS \\
\hline Percentage who are high-school graduates ${ }^{a}$ & 50 & 100 & $P<.001$ \\
\hline
\end{tabular}




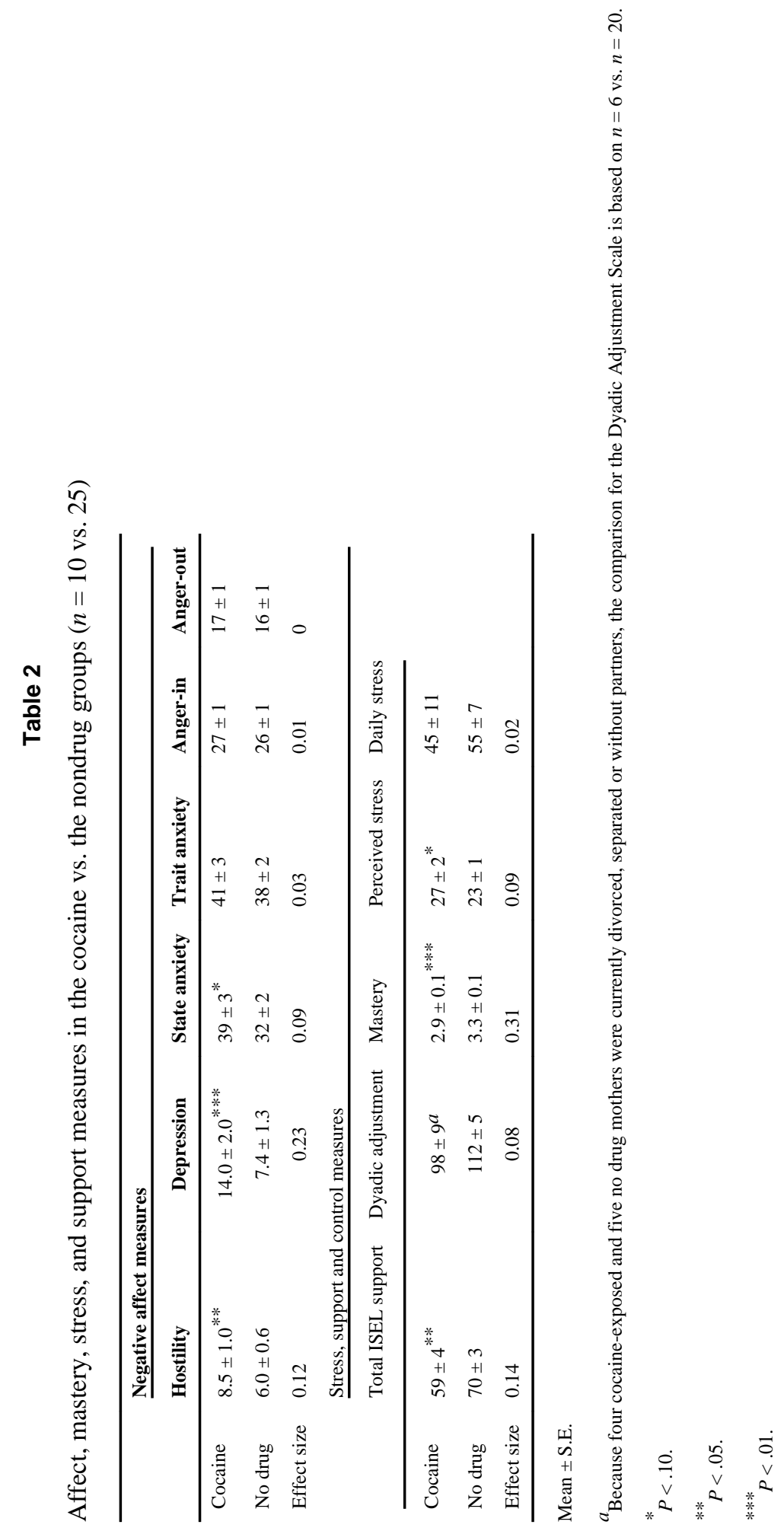

Addict Behav. Author manuscript; available in PMC 2011 June 7. 
Table 3

Oxytocin level during stress after baby contact: correlations with blood pressure and psychosocial measures in the full sample $(N=35)$

\begin{tabular}{cllll}
\hline \multicolumn{3}{c}{ SBP } & DBP & Psychosocial \\
\hline No baby day & & \multicolumn{3}{l}{ Positive state or trait } \\
Base & $-0.32^{*}$ & -0.27 & Mastery & $+0.33^{* *}$ \\
Prep & $-0.29^{*}$ & $-0.42^{* *}$ & Total ISEL support & $+0.40^{* *}$ \\
Speech & -0.09 & -0.11 & Dyadic adjustment & +0.18 \\
Replay & $-0.42^{* *}$ & $-0.41^{* *}$ & & \\
Baby day & & & Negative state or trait & \\
Base & -0.26 & $-0.29^{*}$ & Depression & -0.20 \\
Prep & $-0.29^{*}$ & -0.17 & State anxiety & -0.12 \\
Speech & -0.23 & -0.02 & Trait anxiety & -0.25 \\
Replay & -0.24 & -0.12 & Anger-in & -0.02 \\
Ambulatory & & & Anger-out & -0.11 \\
Wake & -0.22 & -0.17 & Perceived stress & -0.23 \\
Feed & $-0.35^{* *}$ & -0.25 & Daily stress & +0.06 \\
Sleep & $-0.34^{* *}$ & -0.26 & Hostility & -0.11 \\
\hline
\end{tabular}

$\stackrel{*}{P}<.10$.

** $P<.05$. 